\title{
Urethral Stricture Disease: Evaluation of the Male Urethra
}

\author{
Joel Gelman, MD and James Furr, MD
}

\begin{abstract}
The diagnosis of urethral stricture disease is often made when a man with obstructive voiding symptoms undergoes flexible cystoscopy (urethroscopy). However, a more complete and definitive diagnosis is then achieved when the urethra is subsequently evaluated with a retrograde urethrogram, and in many cases, a voiding cystourethrogram (VCUG). In situations where there is stricture disease involving the meatus or fossa navicularis that prevents the passage of adult flexible cystoscope, other testing appropriate in these cases include distal urethral calibration using bougie-a-boules and possibly the advancement of a pediatric cystoscope. It is only after the exact location and length and severity of the stricture is assessed that patients can be properly advised of all options before decisionmaking and the implementation of a treatment plan. This section will review the evaluation of the male urethra.
\end{abstract}

Keywords: urethral stricture, urethra, urethral imaging

Indications

A LTHOUGH EVERY PATIENT EVALUATION begins with a complete history and physical evaluation, there are particular aspects of the history related to patients with suspected or known urethral stricture disease. Obstructive symptoms in a man with a history of hypospadias, especially after reconstructive surgery, are highly suggestive of urethral stricture disease. Moreover, prior instrumentation and catheterization are associated with strictures of the fossa navicularis. ${ }^{1}$ When there is a history of any prior dilation or urethrotomy, obstructive symptoms are likely secondary to urethral stricture recurrence as these endoscopic treatments are seldom curative. ${ }^{2,3}$ When there is any history of straddle trauma, bulbar stricture disease should be suspected. When there is any acquired meatal stenosis or history of adult circumcision for phimosis, balanitis xerotica obliterans also known as Lichen Sclerosis (LS) is often that etiology. However, although all the above are associated with a diagnosis of anterior stricture disease, stricture should also be suspected in less obvious presentations, including obstructive symptoms with a history of prostatitis or epididymitis, or benign prostatic hypertrophy unresponsive to medical management.

In addition, pelvic fracture urethral injures initially managed with suprapubic tube urinary diversion and strictures after prostate surgery, radiation, and/or high intensity focused ultrasound are examples of disorders of the posterior urethra that require evaluation before treatment.

\section{Preoperative Preparation}

In patients who do not have indwelling catheters, a urinalysis with microscopy is indicated before instrumentation and imaging as a negative urinalysis is ideal, and an untreated infection may increase the risk of infectious complications. When there has been instrumentation such as catheterization or dilation during the previous 3 months, any evaluation will likely provide useful general information, but can underestimate the stricture length and severity (Figs. 1 and 2). Therefore, before consideration of open reconstructive surgery, imaging should be performed after a 3-month period of urethral rest. ${ }^{4}$ In some patients with aggressive strictures who are managed with frequent dilations or intermittent catheterization, they cannot discontinue catheterization without high risk of developing retention in the interim. These men will often benefit from having a suprapubic tube placed to allow the bladder to empty as the stricture stabilizes.

\section{Patient Positioning}

The initial evaluation of the urethra with calibration and/or urethroscopy is performed with the patient in the supine position. Urethral imaging is performed with the patient in the oblique position.

\section{Surgical Steps}

Flexible cystoscopy is generally performed with a cystoscope that is $\sim 16 \mathrm{~F}$ in size after the patient is prepped and

\footnotetext{
Department of Urology, University of California, Irvine, Orange, California, USA.
}

(C) Joel Gelman and James Furr 2020; Published by Mary Ann Liebert, Inc. This Open Access article is distributed under the terms of the Creative Commons Attribution Noncommercial License (http://creativecommons.org/licenses/by-nc/4.0/) which permits any noncommercial use, distribution, and reproduction in any medium, provided the original author(s) and the source are cited. 
draped and lidocaine jelly is gently instilled into the urethra. The objective is to assess for the presence or absence of a stricture. As the scope is advanced, once a stricture is encountered where the caliber of the urethra is less than the caliber of the scope, if any attempt is made to advance or manipulate the scope farther proximally, the procedure is no longer a diagnostic test but rather a "scope dilation" treatment. If the objective is to first obtain complete diagnostic information before consideration of treatment, then the scope should be removed. Information obtained is the appearance and caliber of the urethra before the narrow caliber stricture, and the caliber of the stricture at the distal aspect. Information not obtained is the exact length and often the exact location, especially in more proximal strictures.

The retrograde urethrogram (RUG) is then performed. Although fluoroscopy is often used to perform urethral imaging, we prefer $14 \times 17$ inch plain film imaging because of the high resolution, and improved field of view associated with flat plate imaging. Moreover, we have found that although magnification and oblique position in general can affect image size within a film, our flat plate imaging allows accurate length measurements where the stricture length noted on the film with a ruler is the length of the stricture. Traditional flat plate radiographs were developed in a dark room and available only as printed films. However, with current technology, digital plates can be used. Advantages include the ability to see the image on a monitor seconds after the image is captured without moving the plate, the ability of the image to be digitally entered into the medical record system, and the ability of the image to be printed on both $14 \times 17$ film and smaller glossy paper without the need for a dark room.

Although kidney, ureter, and bladder radiograph (KUB) tables are often used for imaging in Imaging Centers, our preference is the use of a portable KUB machine and a gurney. Advantages of this alternative to a fixed KUB table include, in addition to lower cost, a more comfortable surface for the patient, the ability of the upper part to be raised, which can facilitate voiding when a voiding cystourethrogram (VCUG) is performed, and the feature of a side rail. When the patient is positioned, the center of the image should be the

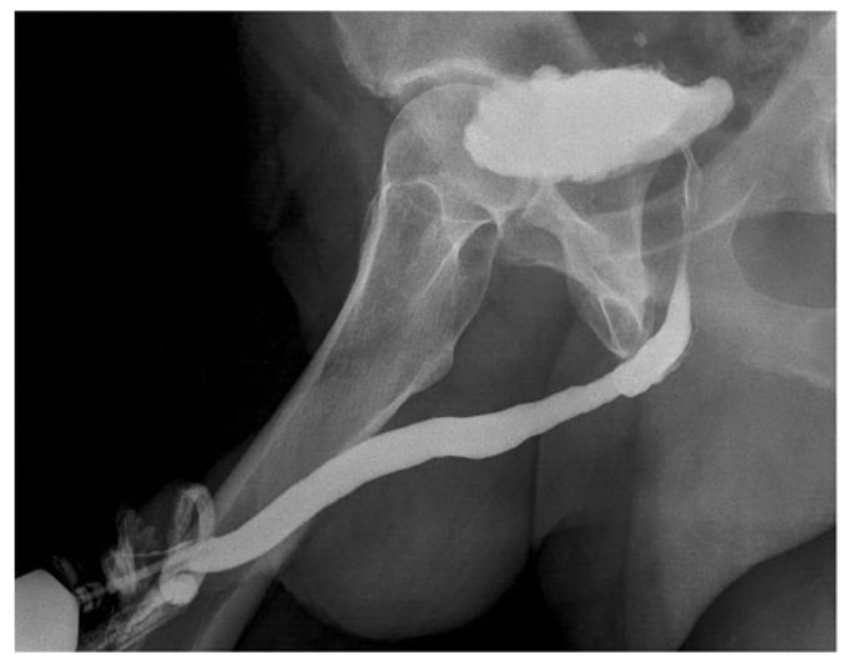

FIG. 1. RUG obtained shortly after discontinuation of catheterization. $\mathrm{RUG}=$ retrograde urethrogram.

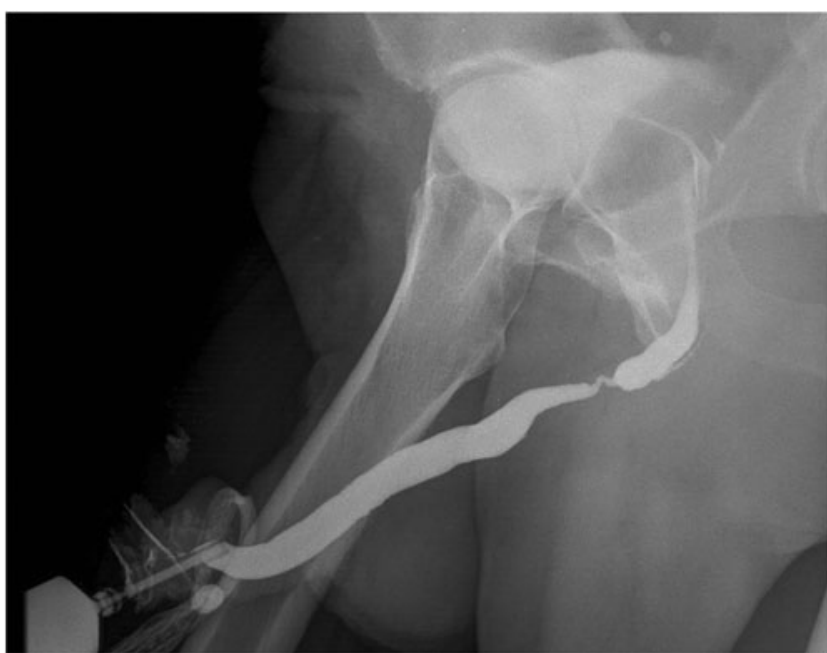

FIG. 2. RUG, same patient, after 3 months of urethral rest subsequent to suprapubic urinary diversion, clearly demonstrating stricture length and location and severity.

base of the penis, and the side of the body that is elevated is positioned against a $45^{\circ}$ spinal wedge that is toward the side of the table or gurney. The use of a side rail secures the wedge, facilitating oblique positioning.

A scout film is the initial image obtained. The purpose of the scout film is to insure proper position and exposure before contrast injection. We see many outside films where a pelvic image with the patient supine is obtained before fluoroscopic images during contrast injection, but this does not fulfill the objectives of a scout film. The injection of contrast is performed as contrast is instilled where the penis is under gentle stretch. Our preferred technique is to use a gauze wrapped around the coronal margin. We wet the gauze with contrast and this provides orientation to the location of the junction of the penile shaft and glans on the film. A tapered adaptor connected to a $60 \mathrm{cc}$ syringe filled with full strength contrast is then gently advanced into the urethral opening, creating a seal, and during the slow advancement of contrast, a film is obtained (Figs. 3 and 4).

It is often desirable to obtain a VCUG, especially when there is any concern about stricture disease involving the membranous urethra, prostatic urethra, and/or bladder neck. When contrast is being injected during a RUG, the entire

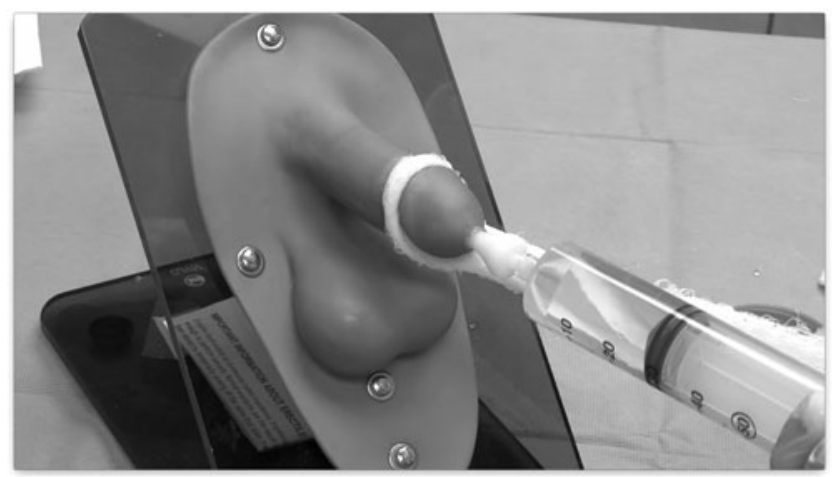

FIG. 3. Technique of RUG. 


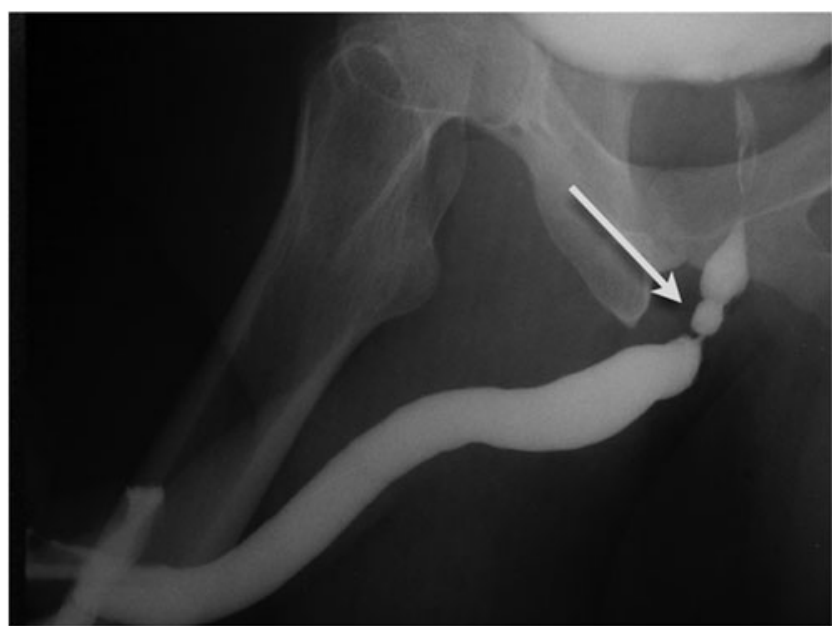

FIG. 4. RUG demonstrating a proximal bulbar stricture (arrow).

posterior urethra is supposed to be coapted and narrow due to the fact that the patient is not voiding at the time, and therefore, sphincters are working to prevent incontinence. Since the posterior urethra will not be widely patent as a RUG is being performed but with and without the presence of stricture or stenosis involving the posterior urethra, the RUG is a poor test of patency of the posterior urethra. However, during voiding, if a patient can relax to void as a film is being obtained, a normal bladder neck and prostatic and membranous urethra will open with wide patency when normal, but there will be constriction when there is an abnormality (Fig. 5). After the RUG is performed, to obtain a VCUG, our approach is to slowly and gently continue to instill contrast in retrograde manner until the patient has a strong sensation to void. Then, during voiding, a film is obtained.

In cases where there is stricture involving the urethral meatus or fossa navicularis, the standard adaptor we use will not advance. Our first step is to calibrate the distal urethra

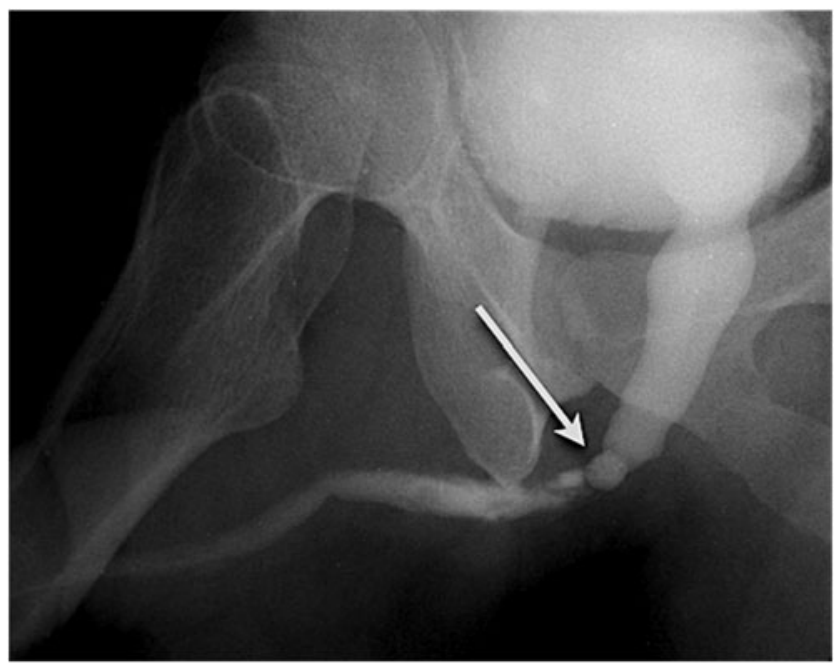

FIG. 5. VCUG not only demonstrating the bulbar stricture (arrow) seen on the RUG in Figure 4 but also demonstrating the wide patency of the posterior urethra. $\mathrm{VCUG}=$ voiding cystourethrogram.

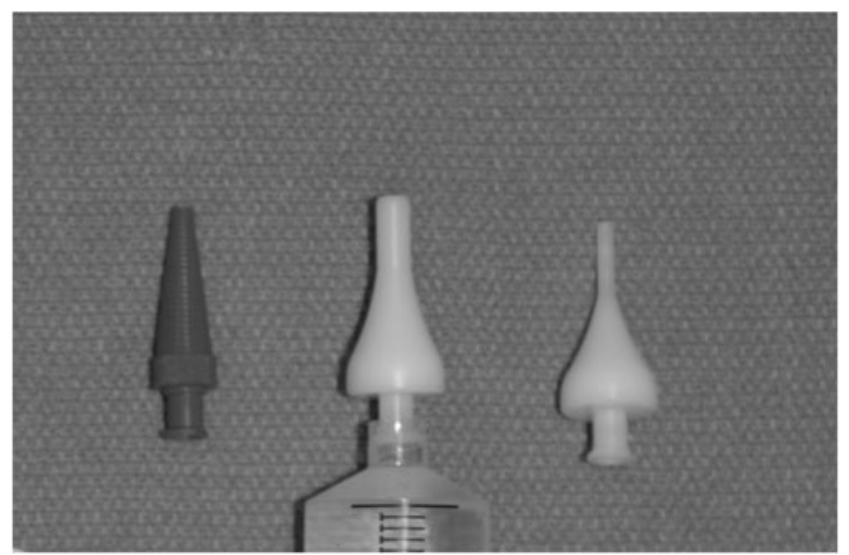

FIG. 6. Christmas tree type adaptor (left), adult Taylor adaptor (center), pediatric Taylor adaptor (right).

using bougie-a-boules along a lubricant such as lidocaine jelly, beginning with a size that will advance and withdraw without any catch or resistance. Then, sequentially larger bougies are advanced with $1.5 \mathrm{~F}$ to $2 \mathrm{~F}$ increments until there is a slight catch as the cone-shaped instrument is withdrawn. This represents the caliber. These instruments, which should not be advanced far into the urethra, provide an extremely precise measurement. When the distal caliber is at least $10 \mathrm{~F}$, we can advance a pediatric version of the Taylor adaptor. Moreover, this permits the advancement of a pediatric cystoscope to evaluate the urethra endoscopically. This is particularly useful in patients who have had prior hypospadias repair as the presence or absence of hair within the reconstructed urethra can be assessed, as the presence of hair may affect the management decision making. When the urethral opening is $<10 \mathrm{~F}$, which is not uncommon in patients with LS, an adaptor such as a "Christmas Tree" adaptor can create a seal at the opening without any advancement into the urethra (Fig. 6).

Patients with pelvic fracture urethral injuries with urethral obliterations will have suprapubic tubes. Evaluation before posterior urethral reconstruction is indicated to assess the exact

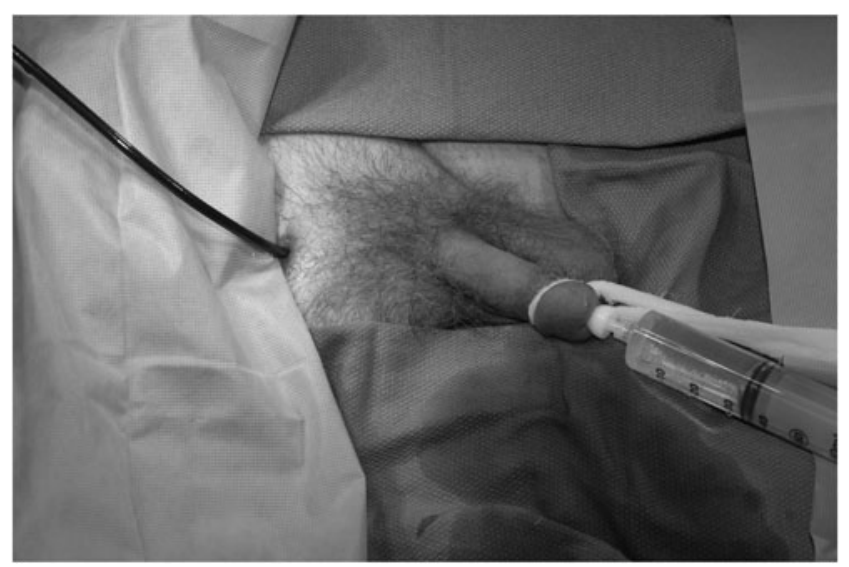

FIG. 7. RUG with simultaneous cystourethrogram obtained with injection of contrast through a flexible cystoscope with the tip at or distal to the verumontanum. 


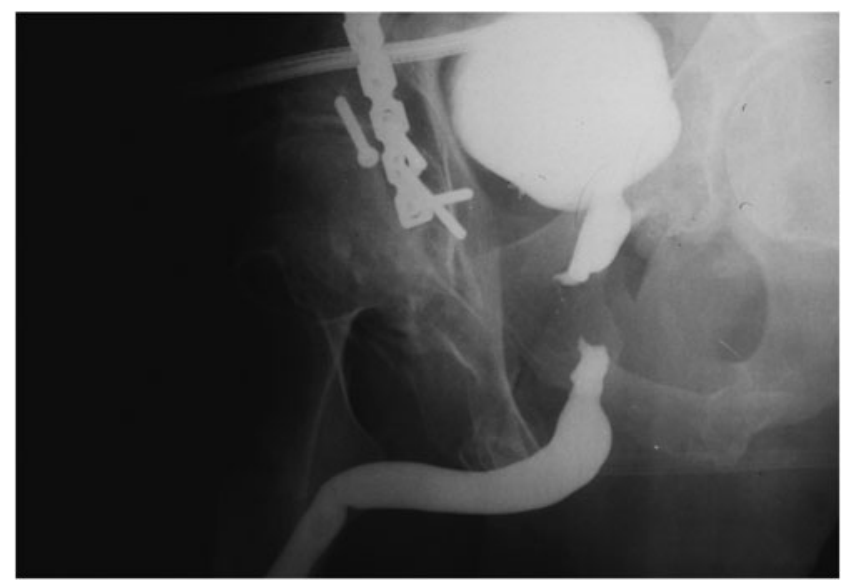

FIG. 8. Image demonstrating the length and location of a pelvic fracture urethral injury.

length and location of the obliteration, which is generally within the distal membranous and most proximal bulbar urethra. ${ }^{5}$ It is ideal that the suprapubic tube be at least $16 \mathrm{~F}$ in caliber, midline, and at least $2 \mathrm{~cm}$ superior to the midline pubic symphysis as this facilitates antegrade cystoscopy in addition to curved sounds at the time of posterior urethroplasty. This evaluation is often performed in the operating room.

With the patient in the oblique position, urethroscopy will evaluate the urethra up to the point of obliteration and confirm that the distal aspect is before the verumontanum. Antegrade cystoscopy will allow direct visualization of the bladder neck to confirm that it is intact, and as the scope is passed through the bladder neck into the prostatic urethra, visualization of the verumontanum with the proximal aspect of the obliteration distal to the verumontanum confirms that the injury is distal to the prostate. With the tip of the scope in the prostatic urethra, contrast is injected as a RUG is performed. The simultaneous RUG and cystourethrogram allows precise assessment of the location and length of the obliteration (Figs. 7 and 8).

In patients with a history of prostate radiation, antegrade cystoscopy is particularly advantageous because radiation

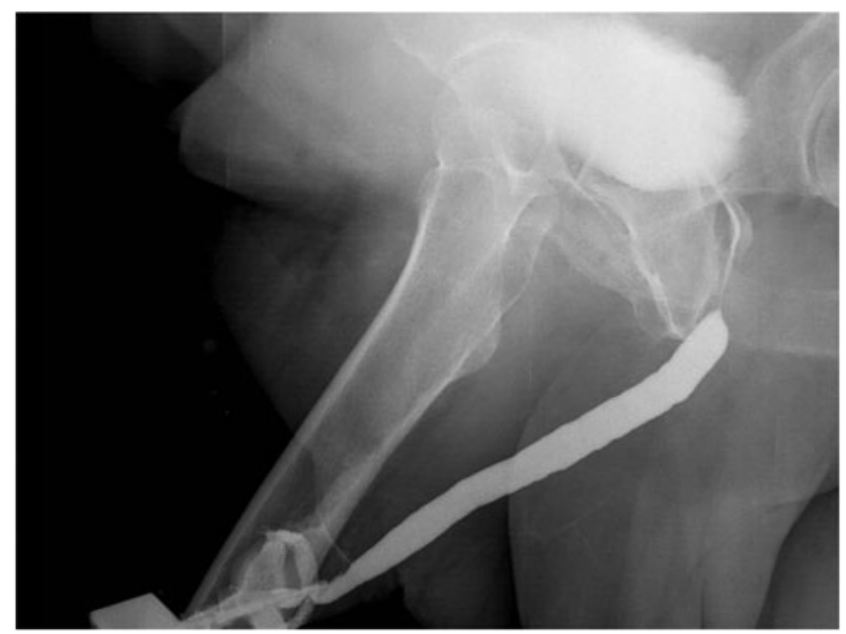

FIG. 9. RUG in a patient with a history of radiation.

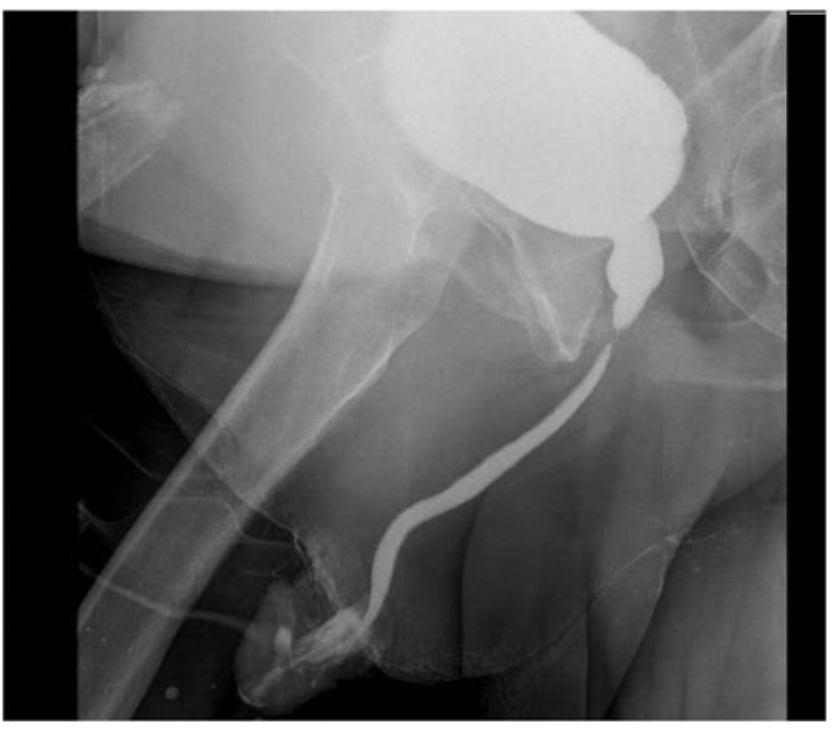

FIG. 10. VCUG demonstrating the membranous radiation stricture and confirming wide patency of the bladder neck and prostatic urethra.

strictures typically involve the membranous urethra and are amenable to urethroplasty with excision and primary anastomosis. However, this repair is best pursued when there is clarity that there is no obvious involvement of the prostatic urethra or bladder neck (Figs. 9 and 10).

\section{Alternative Approaches}

An alternative approach to urethral imaging in patients with anterior strictures, including recurrent strictures is for the patient to be treated with dilation or incision without prior imaging or discussion of urethroplasty as an alternative. It has been our observation that this is by far the most common approach to stricture treatment in our patient population. ${ }^{6}$ However, a disadvantage of this approach is that endoscopic treatments are generally ineffective, and especially ineffective for long or recurrent strictures. Without prior imaging to assess stricture length, the expected outcome of an endoscopic treatment cannot be discussed. Moreover, informed consent is best obtained when all options are discussed, including the option that is associated with a high cure rate. Current American Urological Association guidelines indicate that imaging is generally indicated to evaluate strictures

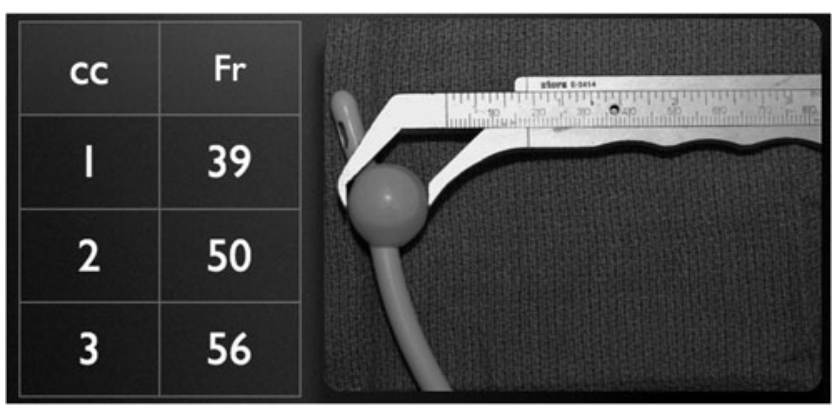

FIG. 11. Balloon inflation with only $2 \mathrm{cc}$ within the fossa navicularis dilates the urethra to $\sim 50 \mathrm{~F}$. 
before treatment, and open repair is indicated for longer or recurrent strictures in particular. ${ }^{7}$

Alternative approaches to our preferred method include a common technique of advancing a catheter in the urethra and inflating the balloon in the fossa navicularis. It is our view that this is a poor technique. The inflation of a balloon with only $2 \mathrm{cc}$ will dilate the urethra to at least $50 \mathrm{~F}$, and the normal fossa navicularis is generally $22 \mathrm{~F}$ to $24 \mathrm{~F}$ (Fig. 11). This is often a painful approach, and we have seen fossa navicularis strictures develop subsequent to imaging with this approach. Moreover, when a catheter is inflated and pulled, to place the penis on stretch, the penis is often not adequately on stretch because of concern about the balloon becoming dislodged. When the penis is not on stretch, the stricture length can be underestimated.

Other alternative techniques to evaluate the urethra include urethral ultrasound and in selected cases, MRI. ${ }^{8-10}$ Ultrasound offers an assessment of the degree of spongiofibrosis, but we have not found ultrasound of value in decision-making. This is an area of controversy. An alternative approach to imaging in patients with pelvic fracture urethral injuries is for the bladder to be filled via the suprapubic tube and for the patient to attempt to void or Valsalva as a RUG is being performed. The limitation of that technique is that when the bladder neck does not open, there is no or incomplete visualization of the posterior urethra proximal to the injury, falsely suggesting an obliteration extending into the prostatic urethra.

\section{Outcomes}

Urethral evaluation and imaging with proper technique is associated with a low complication rate, is well tolerated, and provides definitive diagnostic information regarding the presence or absence of stricture and when there is a stricture diagnosed, imaging accurately assesses stricture length, location, and severity. Urethroplasty outcomes include success rates that can exceed $98 \%$ with excision and primary anastomosis. ${ }^{11,12}$ However, excisional repair is a preferred approach for shorter bulbar strictures in particular, and this is an example of how proper imaging is important to guide decision-making.

\section{Author Disclosure Statement}

No competing financial interests exist.

\section{Funding Information}

No funding was received for this article.

\section{Supplementary Data}

Supplementary Video S1

\section{References}

1. Yee DS, Ahlering TE, Gelman J, Skarecky DW. Fossa navicularis strictures due to $22 \mathrm{~F}$ catheters used in robotic radical prostatectomy. JSLS 2007;11:321-325.
2. Naude AM, Heyns CF. What is the place of internal urethrotomy in the treatment of urethral stricture disease? Nat Clin Pract Urol 2005;2:538-545.

3. Heyns CF, Steenkamp JW, De Kock ML, Whitaker P. Treatment of male urethral strictures: Is repeated dilation or internal urethrotomy useful? J Urol 1998;160:356-358.

4. Terlecki RP, Steele MC, Valadez C, Morey AF. Urethral rest: Role and rationale in preparation for anterior urethroplasty. Urology 2011;77:1477-1481.

5. Latini JM, McAninch JW, Brandes SB, Chung JY, Rosenstein D. SIU/ICUD Consultation On Urethral Strictures: Epidemiology, etiology, anatomy, and nomenclature of urethral stenoses, strictures, and pelvic fracture urethral disruption injuries. Urology 2013;83:S1-S7.

6. Wisenbaugh ES, De Grado JJ, Quinn R, Gelman J. Men with anterior urethral strictures are often treated without imaging or discussion of all treatment options. Urol Pract 2017;5:52-56.

7. Wessells H, Angermeier KW, Elliot SP, et al. Male Urethral Stricture. American Urological Association (AUA) Guideline 2016:1-34. https://www.auanet.org/guidelines/ urethral-stricture-guideline

8. Morey AF, McAninch JW. Sonographic staging of anterior urethral strictures. J Urol 2000;163:1070-1075.

9. Chen L, Feng C, Lv XG, et al. Three-dimensional computerized model based on the sonourethrogram: A novel technique to evaluate anterior urethral stricture. J Urol 2018;199:568-575.

10. Oh MM, Jin MH, Sung DJ, Yoon DK, Kim JJ, Moon du G. Magnetic resonance urethrography to assess obliterative posterior urethral stricture: Comparison to conventional retrograde urethrography with voiding cystourethrography. J Urol 2010;183:603-607.

11. Santucci RA, Mario LA, McAninch JW. Anastomotic urethroplasty for bulbar urethral stricture: Analysis of 168 patients. J Urol 2002;167:1715-1719.

12. Eltahawy EA, Virasoro R, Schlossberg SM, McCammon $\mathrm{KA}$, Jordan GH. Long-term followup for excision and primary anastomosis for anterior urethral strictures. J Urol 2007; 177:1803-1806.

Address correspondence to: Joel Gelman, MD

Department of Urology University of California, Irvine

333 City Blvd. W \#2100

Orange, CA 92868

USA

E-mail: jgelman@uci.edu

$$
\begin{aligned}
\text { Abbreviations Used } & \\
\text { KUB } & =\text { kidney, ureter, and bladder radiograph } \\
\text { LS } & =\text { Lichen } \text { Sclerosis } \\
\mathrm{MRI} & =\text { magnetic resonance imaging } \\
\mathrm{RUG} & =\text { retrograde urethrogram } \\
\mathrm{VCUG} & =\text { voiding cystourethrogram }
\end{aligned}
$$

